\title{
Inference and Sample Size Calculations Based on Statistical Tests in a Negative Binomial Distribution for Differential Gene Expression in RNA- seq Data
}

\author{
Raiaoh \\ ${ }^{1}$ Department of Bioinformatics and Biostatistics, University of Louisville, Louisville, KY, 40202, USA \\ ${ }^{2}$ Department of Anatomical Sciences and Neurobiology, University of Louisville, Louisville, KY, 40202, USA \\ ${ }^{3}$ Department of Biostatistics, Vanderbilt University, Nashville, TN, 37232, USA \\ ${ }^{4}$ Department of Computer Engineering and Computer Science, University of Louisville, Louisville, KY, 40292, USA \\ ${ }^{5}$ Department of Mathematics, University of Louisville, Louisville, KY, 40292, USA \\ ${ }^{6}$ Department of Cardiology, University of Louisville, Louisville, KY, 40202, USA \\ ${ }^{7}$ Department of Biomedical Informatics, Ohio State University, Columbus, OH, 43210, USA
}

\begin{abstract}
The high throughput RNA sequencing (RNA-seq) technology has become the popular method of choice for transcriptomics and the detection of differentially expressed genes. Sample size calculations for RNA-seq experimental design are an important consideration in biological research and clinical trials. Currently, the sample size formulas derived from the Wald and the likelihood ratio statistical tests with a Poisson distribution to model RNA-seq data have been developed. However, since the mean read counts in the real RNA-seq data are not equal to the variance, an extended method to calculate sample sizes based on a negative binomial distribution using an exact test statistic was proposed by Li et al. in 2013. In this study, we alternatively derive five sample size calculation methods based on the negative binomial distribution using the Wald test, the log-transformed Wald test and the log-likelihood ratio test statistics. A comparison of our five methods and an existing method was performed by calculating the sample sizes and the simulated power in different scenarios. We first calculated the sample sizes for testing a single gene using the six methods given a nominal significance level $\alpha$ at 0.05 and $80 \%$ power. Then, we calculated the sample sizes for testing multiple genes given a false discovery rate (FDR) at 0.05 and 0.10 . The empirical power and true prognostic genes for differential gene expression analysis corresponding to the estimated sample sizes from the six methods are also estimated via the simulation studies. Using the sample size formulas derived from log-transformed and Wald-based tests, we observed smaller sample properties while maintaining the nominal power close to or higher than $80 \%$ in all the settings compared to other methods. Moreover, the Wald test based sample size calculation method is easier to compute and faster in an RNA-seq experimental design.
\end{abstract}

Keywords: FDR; Sample size; Wald test; Exact test; Likelihood ratio test

\section{Introduction}

Sample size calculations are a prerequisite in an experimental design for biological research and clinical trials. Recently, highthroughput RNA sequencing (RNA-seq) technology has been widely used for gene expression studies in a variety of applications, such as expression profiling of mRNAs or non-coding RNA [1-3], de novo assembly and characterization of transcriptomes [4,5], and the identification of novel alternatively spliced transcript [6,7]. These novel transcripts or differentially expressed genes (DEGs) identified from RNA-seq data may serve as human disease biomarkers or gene signatures for the clinical diagnosis [8-10]. With the rapid growth of RNA-seq applications, sample size calculation methods derived from test statistics with an appropriate distribution are important issues to be explored and discussed.

Due to the initial high cost of RNA-seq, sample size, in terms of the number of biological replicates, was not seriously considered as part of the experimental design. As a case in point, one RNA-seq review article [11] documented several RNA-seq studies showing that many had only one or a few biological replicates. While thousands of DEGs were identified within these studies, the lack of biological replicates leads to an absence of knowledge concerning biological variations and may result in a high percentage of false positive genes. Therefore, ignorance of biological variation is the fundamental problem with the analyzed results collected from un-replicated data. A recent paper [12] was the first to point out that conclusions drawn from un-replicated samples can be misleading and unrealistic. Later, another study [13] further addressed design and validation issues due to the lack of biological replicates in RNA-seq data.

One of the key questions in an experimental design is to determine the number of biological replicates needed for differential expression analysis in order to achieve a desired statistical power given a significance level $\alpha$ and an underlying distribution. Since RNA-seq data are read counts, a Poisson distribution is commonly used as the model for identifying DEGs in RNA-seq data [14,15]. Fang and Cui were the first one to derive the sample size calculations based on a Wald statistical test with a Poisson distribution for single gene in RNA-seq.

*Corresponding author: Rai SN, Department of Bioinformatics and Biostatistics, University of Louisville Louisville, KY, 40202, USA, Tel: 1-502-852-4030; E-mail: Shesh.Rai@louisville.edu

Received December 21, 2016; Accepted January 25, 2017; Published January 30, 2017

Citation: Li X, Cooper NGF, Shyr Y, Wu D, Rouchka EC, et al. (2017) Inference and Sample Size Calculations Based on Statistical Tests in a Negative Binomial Distribution for Differential Gene Expression in RNA-seq Data. J Biom Biostat 8 : 332. doi:10.4172/2155-6180.1000332

Copyright: ( $2017 \mathrm{Li} \mathrm{X}$, et al. This is an open-access article distributed under the terms of the Creative Commons Attribution License, which permits unrestricted use, distribution, and reproduction in any medium, provided the original author and source are credited. 
Later, several sample size calculation methods that were derived from the score statistic and the log-likelihood ratio test (LRT) statistic using the Poisson distribution were proposed [16]. However, the assumption of a Poisson distribution that the expected mean and variance are equal usually does not hold for RNA-seq studies, where the variance is typically greater than the mean of the read counts [17]. Therefore, a negative binomial distribution with a dispersion parameter is used to model RNA-seq data by the existing software packages such as DESeq [17] and edgeR [18], in which an exact test is used to test DEGs between conditions. Subsequently, a sample size calculation method based on an exact test statistic with the aid of the edgeR package [18] was proposed [19]. However, sample size methods derived from other test statistics such as the Wald test, the LRT and an extension of Wald test via log-transformation using negative binomial distribution to model the RNA-seq data have not yet been explored.

Like microarray data, an RNA-seq dataset contains thousands of genes to be tested simultaneously and independently for differential expression analysis. A method for the adjustment or correction of $\mathrm{p}$-values is required to control the type I error rate when multiple pairwise comparisons are performed. Instead of setting the critical value $\alpha$ at 0.05 or 0.01 for significance, a much lower critical value $\alpha^{*}$ is required to correct for the inflation of $a$. The most common method to control the family-wise error rate (FWER) is the Bonferroni correction in which the adjusted p-value is computed via dividing the critical p-value by the total number of comparisons being made. The other widely used method for this multiple correction problem is an FDR correction [20]. Since the Bonferroni correction with a large number of tests is more conservative than the FDR correction, using the Bonferroni correction results in a cost of increasing the probability of producing false negatives and consequently reducing the statistical power. For high dimensional microarray data analysis, an extension of the FDR correction was proposed [21] and is widely used by many researchers. To address similar issues for high-dimensional RNA-seq data, a sample size determination based on the extended FDR correction from microarray data analysis was further proposed [16].

Our study is motivated by exploring sample size calculations using the well-known test statistics (the Wald test and LRT) and a negative binomial distribution to model RNA-seq data. In Section 2, we first define the Wald test, the log-transformed Wald test and the LRT statistics using the negative binomial distribution to model a single gene in RNA-seq data. Then, we derive sample size calculations based on these defined statistical tests. Lastly, we derived sample size calculation methods for testing multiple genes while controlling the FDR [16]. In Section 3, we simulated power for testing single gene and multiple genes corresponding to the sample sizes estimated from our proposed methods and an existing method. The performance of these six methods is compared and evaluated via the required sample sizes and the estimated power. An application of real RNA-seq data to illustrate sample size calculations is presented in Section 4. Finally, we end with a discussion and conclusion in Section 5.

\section{Methods}

\section{Derivation of test statistics}

In an RNA-seq experiment, the data contains thousands of genes $(g=1, \ldots, G)$ with different number of reads for each sample mapped to the reference genome. Since the total number of reads among samples is different, the distribution of the gene in the sample with the same condition is not identical. A normalization factor called the size factor is used to model RNA-seq data with a negative binomial distribution. For simplicity, the following statistical tests and consequent sample size calculation methods are based on a single gene tests for DEG analysis.

For a single gene in RNA-seq data, suppose that, for each condition $i(\mathrm{i}=0,1)$, the observations $X_{i j}\left(j=1, \ldots, n_{i}\right)$, are independent and identically follow a negative binomial distribution as $X_{i j} \sim \mathrm{NB}\left(s_{\mathrm{ij}} \gamma_{\mathrm{i}}, \phi\right)[17,22]$. Under this setting, $\gamma_{\mathrm{i}}$ is the true gene expression level in condition $i, s_{i j}$ is a size factor to normalize the raw read for the total number of reads mapped in the sample $j$, and $\phi$ is a dispersion parameter with the assumption $\phi=\phi$. Thus, the summation of reads per gene per condition $\left(X_{i}=\sum_{j=1}^{n_{i}} X_{i j}\right)$ also follows a negative binomial distribution with parameters nu $u_{\mathrm{i}}=\mathrm{t}_{\mathrm{i}}=\mathrm{s}_{\mathrm{i}} \gamma_{\mathrm{i}}$ and $\oint / n$, where $s_{i}=\sum_{j=1}^{n_{i}} s_{i j}$ is the summation of the size factor for mapping reads in condition $i$ and $n$ is the number of biological replicates with the assumption $n_{i}=n$.

For detection of a differentially expressed gene from RNA-seq data, the ratio $\gamma_{1} / \gamma_{0}$ typically represents the fold change $\left(\rho=\gamma_{1} / \gamma_{0}\right)$. If the fold change equals one, we can say that this gene is not differentially expressed. Therefore, we are interested in making an inference about the ratio using the Wald statistics and the likelihood ratio methods for sample size calculations.

For testing the hypothesis about the fold change in regards to the DEGs, it is equivalent to test the hypothesis,

$H_{0}: \gamma_{1}=\gamma_{0}$ vs. $H_{1}: \gamma_{1} \neq \gamma_{0}$.

Since $\gamma_{\mathrm{i}}(\mathrm{i}=0,1)$ and $\oint$ are unknown parameters, we use the following two sample estimates for these parameters under the negative binomial distribution in RNA-seq data [23].

\section{Unconstrained maximum likelihood estimate (MLE)}

The likelihood and $\log$-likelihood function of $X_{0} \sim N B\left(s_{0} \gamma_{0}, \frac{\phi}{n}\right)$ and $X_{1} \sim N B\left(s_{1} \gamma_{1}, \frac{\phi}{n}\right)$ are:

$$
\begin{aligned}
& \mathrm{L}\left(\gamma_{0}, \gamma_{1}, \phi \mid x_{0}, x_{1}\right)=\prod_{i=0}^{1} \frac{\Gamma\left(\frac{n}{\phi}+x_{i}\right)}{\Gamma\left(\frac{n}{\phi}\right) \cdot x_{i} !}\left(\frac{s_{i} \gamma_{i} \phi / n}{\left(s_{i} \gamma_{i} \phi / n\right)+1}\right)^{x_{i}}\left(\frac{1}{\left(s_{i} \gamma_{i} \phi / n\right)+1}\right)^{\frac{n}{\phi}}, \\
& \operatorname{lnL}\left(\gamma_{0}, \gamma_{1}, \phi \mid x_{0}, x_{1}\right)=\sum_{i=0}^{1} \ln \Gamma\left(\frac{n}{\phi}+x_{i}\right)-2 \ln \Gamma\left(\frac{n}{\phi}\right)-\sum_{i=0}^{1} \ln x_{i} !+\sum_{i=0}^{1} x_{i} \ln \left(s_{i}\right)+\sum_{i=0}^{1} x_{i} \ln \left(\gamma_{i}\right)+\sum_{i=0}^{1} x_{i} \ln \left(\frac{\phi}{n}\right)-\sum_{i=0}^{1}\left(x_{i}+\frac{n}{\phi}\right) \ln \left(\frac{s_{i} \gamma_{i} \phi}{n}+1\right),
\end{aligned}
$$


where $t_{0}=n u_{0}=s_{0} \gamma_{0}$ and $\mathrm{t}_{1}=\mathrm{nu}_{1}=\mathrm{s}_{1} \gamma_{1}$. Setting the first derivative to zero, we obtain the unrestricted MLEs with respect to $\gamma_{i}: \hat{\gamma}_{0}=\frac{x_{0}}{s_{0}}$ and $\hat{\gamma}_{1}=\frac{x_{1}}{s_{1}}$. Subsequently, we can obtain the
MLE of $\hat{\phi}$ by solving the following equation:

$$
\frac{\partial l}{\partial \phi}=\sum_{i=0}^{1} \varnothing\left(\frac{n}{\phi}+x_{i}\right)-2 \varnothing\left(\frac{n}{\phi}\right)+\frac{n}{\phi^{2}}\left\{\ln \left(\bar{x}_{0} \phi+1\right)+\ln \left(\bar{x}_{1} \phi+1\right)\right\}-\frac{\bar{x}_{0}\left(x_{0}+\frac{n}{\phi}\right)}{\left(\bar{x}_{0} \phi+1\right)}-\frac{\bar{x}_{1}\left(x_{1}+\frac{n}{\phi}\right)}{\left(\bar{x}_{1} \phi+1\right)}=0,
$$

Several mathematical optimization methods, such as the Newton-Raphson method, can be used to estimate $\hat{\phi}$ for equation (4). Since there is no closing form to estimate the dispersion $\phi$, we derived the sample size formula based on a constant value of $\phi$ estimated from the data.

\section{Constrained maximum likelihood estimate (CMLE) for $\gamma_{i}$ and $\phi$}

The parameters are estimyated under the null hypothesis $H_{0}: \gamma_{0}=\gamma_{1}$.

Let $w=\frac{s_{1}}{s_{0}}, \rho=\frac{\gamma_{1}}{\gamma_{0}}=\frac{s_{1}}{t_{0}}$ and $\mathrm{t}_{1}=\rho \mathrm{st}_{0}=\rho \mathrm{wnu}_{0}$. Then, the log likelihood function from equation $(2)$ is re-parameterized as:

$\ln \mathrm{L}\left(\rho, t_{0}, \phi \mid x_{0}, x_{1}\right)=\sum_{i=0}^{1} \ln \Gamma\left(\frac{n}{\phi}+x_{i}\right)-2 \ln \Gamma\left(\frac{n}{\phi}\right)-\sum_{i=0}^{1} \ln x_{i} !+x_{0} \ln t_{0}+x_{0} \ln (\phi)+x_{0} \ln \left(\frac{1}{n}\right)+x_{1} \ln \left(t_{0}\right)$

$+x_{1} \ln (w / n)+x_{1} \ln (\phi)+x_{1} \ln (\rho)-x_{0} \ln \left(\frac{t_{0} \phi}{n}+1\right)-x_{1} \ln \left(\frac{\rho w t_{0} \phi}{n}+1\right)-\frac{n}{\phi} \ln \left(\frac{t_{0} \phi}{n}+1\right)-\frac{n}{\phi} \ln \left(\frac{\rho w t_{0} \phi}{n}+1\right)$

Using partial derivatives of the equation (5) with respect to $\rho$ and $t_{0}$, the MLEs in the unrestricted parameter space are $\hat{t}_{0}=x_{0}$ and $\hat{\rho}=\frac{x_{1}}{w x_{0}}$. However, the CMLE $t_{0}$ in the constrained parameter space and under $H_{0}: \rho=1$ is given by

$$
\frac{\partial l\left(\rho=1, t_{0}, \phi \mid x_{0}, x_{1}\right)}{\partial t_{0}}=\frac{x_{0}-t_{0}}{t_{0}\left(\frac{\phi t_{0}}{n}+1\right)}+\frac{x_{1}-w t_{0}}{t_{0}\left(\frac{w \phi t_{0}}{n}+1\right)} .
$$

Setting equation (6) to zero, we obtain:

$$
t_{0}=\frac{\sqrt{\left[\phi\left(w x_{0}+x_{1}\right)-n(1+w)\right]^{2}+8 w \phi n\left(x_{0}+x_{1}\right)}}{4 w \phi}+\frac{\left[\phi\left(w x_{0}+x_{1}\right)-n(1+w)\right]}{4 w \phi} .
$$

Thus, setting the derivative of $\phi$ to the zero and $t_{0}=\tilde{t}_{0}$ and $\rho=1$, the CMLE $\tilde{\phi}$ for $\phi$ is estimated by solving the following equation:

$$
\begin{aligned}
& 0=\sum_{i=0}^{1} \Psi\left(\frac{n}{\tilde{\phi}}+x_{i}\right)-2 \Psi\left(\frac{n}{\tilde{\phi}}\right)+\frac{x_{0}-\tilde{t}_{0}}{\tilde{\phi}\left(\frac{\tilde{t} \tilde{\phi}}{n}+1\right)}+\frac{x_{1}-w \tilde{t}_{0}}{\tilde{\phi}\left(\frac{w \tilde{t}_{0} \tilde{\phi}}{n}+1\right)}+\frac{n}{\tilde{\phi}^{2}}\left\{\ln \left(\frac{\tilde{t}_{0} \tilde{\phi}}{n}+1\right)+\ln \left(\frac{w \tilde{t}_{0} \tilde{\phi}}{n}+1\right)\right\} . \\
& \text { We finally obtain }
\end{aligned}
$$

$$
\begin{aligned}
& \tilde{\gamma}_{1}=\tilde{\gamma}_{0}=\tilde{t}_{0} / s_{0}=\frac{\sqrt{\left[\phi\left(w x_{0}+x_{1}\right)-n(1+w)\right]^{2}+8 w \phi n\left(x_{0}+x_{1}\right)}}{4 w s_{0} \phi}+\frac{\left[\phi\left(w x_{0}+x_{1}\right)-n(1+w)\right]}{4 w s_{0} \phi}, \\
& \text { and } \tilde{u}_{0}=\frac{\tilde{t}_{0}}{n}=\frac{\sqrt{\left[\phi\left(w \bar{x}_{0}+\bar{x}_{1}\right)-(1+\rho w)\right]^{2}+8 w \phi\left(\bar{x}_{0}+\bar{x}_{1}\right)}}{4 w \phi}+\frac{\left[\phi\left(w \bar{x}_{0}+\bar{x}_{1}\right)-(1+w)\right]}{4 w \phi} .
\end{aligned}
$$

Since there is no closing form to estimate the dispersion $\phi$ from MLE and CMLE, we derived the sample size formula based on a fixed and constant value. For simplicity, we set the dispersion in MLE and CMLE to be equal with a combination of fixed dispersion $(0.1,0.5$ and 1$)$.

\section{Wald statistical test and log-transformed Wald statistical test}

Wald statistical test: The Wald statistical test is an asymptotic test based on the normal approximation, which utilizes the large-sample properties of the MLE. Following procedures from the studies [24-26] for comparing two independent Poisson rates with unequal sample frames, we derived the Wald's inference procedures using the properties of $\hat{\gamma}_{i}$ and $\hat{\phi}$ estimated from MLE and $\hat{\gamma}_{i}$ and $\hat{\phi}$ from CMLE with a negative binomial distribution in two conditions $\left(\hat{\gamma}_{i}=\frac{X_{i}}{s_{i}}\right)$, where $\mathrm{X}_{0}$ and $\mathrm{X}_{1}$ in two conditions are assumed to be independent. For simplicity, we set $\hat{\phi}=\tilde{\phi}=\phi$ with a constant for the sample size and power analysis.

The null hypothesis $\mathrm{H}_{0}$ in equation (1) is equivalent to $\mathrm{H}_{0}: \gamma_{1}-\gamma_{0}=0$, and consequently we make inferences based on the quantity $T=\hat{\gamma}_{1}-\hat{\gamma}_{0}=\frac{X_{1}}{s_{1}}-\frac{X_{0}}{s_{0}}$. In this case, the variance of $T$ is $\sigma_{T}^{2}=\frac{\gamma_{1}}{s_{1}}+\frac{\gamma_{0}}{s_{0}}+\frac{\phi}{n}\left(\gamma_{1}^{2}+\gamma_{0}^{2}\right)$ and can be estimated by $s_{T}^{2}=\frac{\hat{\gamma}_{1}}{s_{1}}+\frac{\hat{\gamma}_{0}}{s_{0}}+\frac{\phi}{n}\left(\hat{\gamma}_{1}^{2}+\hat{\gamma}_{0}^{2}\right)$, where the 
parameters are estimated form MLE. Thus, Wald statistical test from MLE can be obtained by the statistic T/S : $^{\circ}$

$$
Z_{w 1}=\frac{X_{1}-w X_{0}}{\sqrt{X_{1}+w^{2} X_{0}+\frac{\hat{\phi}}{n}\left(X_{1}^{2}+w^{2} X_{0}^{2}\right)}},
$$

where $w=\frac{s_{1}}{s_{0}}$, is the ratio of total size factors between the two conditions.

Similarly, for $T=\frac{X_{1}}{s_{1}}-\frac{X_{0}}{s_{0}}, \sigma_{T}^{2}$ can be estimated by $s_{T}^{2}=\frac{\tilde{\gamma}_{1}}{s_{1}}+\frac{\tilde{\gamma}_{0}}{s_{0}}+\frac{\tilde{\phi}}{n}\left(\tilde{\gamma}_{1}^{2}+\tilde{\gamma}_{0}^{2}\right)$ using the parameters estimated from CMLE. By substituting $\tilde{\gamma}_{i}=\frac{n u_{0}}{s_{0}}$ from equation (8-9), the 2 nd Wald statistical test can be obtained by the statistic $\mathrm{T} / \mathrm{S}_{\mathrm{T}}$ :

$$
Z_{w 2}=\frac{X_{1}-w X_{0}}{\sqrt{n w^{2} \tilde{u}_{0}\left(1 / w+1+2 \tilde{\phi} \tilde{u}_{0}\right)}} .
$$

Log-transformation of Wald's statistical test: To test the null hypothesis, it is also equivalent to test $H_{0}: \ln \left(\frac{\gamma_{1}}{\gamma_{0}}\right)=0$. The logarithmic transformation is usually adopted for skewness correction and variance stabilization as suggested by these studies [16,24]. The statistical inference on the quantity is $U=\ln \left(\hat{\gamma}_{1} / \hat{\gamma}_{0}\right)=\ln \left(\frac{X_{1}}{s_{1}}\right)-\ln \left(\frac{X_{0}}{s_{0}}\right)$. Since $\frac{X_{1}}{s_{1}}$ and $\frac{X_{0}}{s_{0}}$ have asymptotically normal distributions, $N\left(\gamma_{1}, \frac{\gamma_{1}}{s_{1}}+\frac{\phi}{n} \gamma_{1}^{2}\right)$ and $N\left(\gamma_{0}, \frac{\gamma_{0}}{s_{0}}+\frac{\phi}{n} \gamma_{0}^{2}\right)$, respectively, $\ln \frac{X_{1}}{s_{1}}$ and $\ln \frac{X_{0}}{s_{0}}$ correspondingly also have an asymptotically normal $N\left(\ln \gamma_{1}, \frac{1}{s_{1} \gamma_{1}}+\frac{\phi}{n}\right)$ and $N\left(\ln \gamma_{0}, \frac{1}{s_{0} \gamma_{0}}+\frac{\phi}{n}\right)$ by the Delta Method and Slutsky's theorem. Thus, the variance of $U$ is $\sigma_{U}^{2}=\operatorname{Var}(U)=\operatorname{Var}\left[\ln \left(\frac{X_{1}}{S_{1}}\right)-\ln \left(\frac{X_{0}}{s_{0}}\right)\right]=\frac{1}{s_{1} \gamma_{1}}+\frac{1}{s_{0} \gamma_{0}}+2 \frac{\Phi}{n}$. U/S $\mathrm{S}_{U}$ can be used for testing $\mathrm{H}_{0}$, when $s_{U}^{2}=\frac{1}{s_{1} \hat{\gamma}_{1}}+\frac{1}{s_{0} \hat{\gamma}_{0}}+2 \frac{\Phi}{n}$ using the parameters estimated from MLEs. Thus, a log-transformation of $\mathrm{Z}_{\mathrm{w} 1}$ is applied and the
test statistic is defined as

$$
Z_{l w 1}=\frac{\ln \left(\frac{X_{1}}{X_{0}}\right)-\ln w}{\sqrt{1 / X_{1}+\frac{1}{X_{0}}+2 \frac{\hat{\phi}}{n}}} \text {, where } \hat{\gamma}_{i}=\frac{X_{i}}{s_{i}} \text { and } \phi=\hat{\phi} \text {. }
$$

Similarly, $\mathrm{U} / \mathrm{S}_{\mathrm{U}}$ can be used for testing $\mathrm{H}_{0}$, when $S_{U}^{2}=\frac{1}{S_{1} \tilde{\gamma}_{1}}+\frac{1}{S_{0} \tilde{\gamma}_{0}}+2 \frac{\Phi}{n}$ using the parameters estimated from CMLE and then, we apply the $\log$-transformation of $\mathrm{Z}_{\mathrm{w} 2}$ and use the test statistic:

$$
Z_{l w 2}=\frac{\ln \left(\frac{X_{1}}{X_{0}}\right)-\ln w}{\sqrt{\frac{1}{n \tilde{u}_{0}}\left(\frac{1}{w}+1+2 \tilde{u}_{0} \tilde{\phi}\right)}} \text {, where } \tilde{\gamma}_{1}=\tilde{\gamma}_{0}=\frac{\tilde{t}_{0}}{s_{0}}=\frac{n \tilde{u}_{0}}{s_{0}} \text {. }
$$

We note that the equations defined in (12) and (13) do not exist when $\mathrm{X}_{0}=0$ or $\mathrm{X}_{1}=0$. In this case, $\mathrm{X}_{0}$ or $\mathrm{X}_{1}$ was adjusted to 0.5 [24,25].

\section{Generalized likelihood ratio test (GLRT)}

The GLRT statistic is defined as the ratio of the maximum value of the likelihood function under the restriction of the null hypothesis to the maximum likelihood function under the unrestricted parameter space. For a vector of parameters $\theta \in \Theta$, the GLRT forH $\mathrm{H}_{0}: \theta \in \Theta_{0}$ versus $\mathrm{H}_{1}: \theta \in \Theta_{1}$ is expressed as

$$
\lambda\left\{x_{0}, x_{1} \mid \theta\left(\gamma_{i}, \phi\right)\right\}=\frac{\sup \left\{L\left(\theta\left(\gamma_{i}, \phi\right) \mid x_{0}, x_{1}\right): \theta \in \boldsymbol{\Theta}_{0}\right\}}{\sup \left\{L\left(\theta\left(\gamma_{i}, \phi\right) \mid x_{0}, x_{1}\right): \theta \in \boldsymbol{\Theta}\right\}}
$$

Where $L\left(\theta\left(\gamma_{i}, \phi\right) \mid x_{0}, x_{1}\right)$ is the likelihood function defined. The denominato $\sup \left\{L\left(\theta\left(\gamma_{i}, \phi\right) \mid x_{0}, x_{1}\right): \theta \in \Theta\right\}$ in equation (14) is obtained using the MLE of $\theta$, where $\hat{\gamma}_{0}=\frac{X_{0}}{s_{0}}$ and $\hat{\gamma}_{1}=\frac{X_{1}}{s_{1}}$. The numerator $\sup \left\{L\left(\theta\left(\gamma_{i}, \phi\right) \mid x_{0}, x_{1}\right): \theta \in \Theta_{0}\right\}$ is obtained using the CMLE of $\theta$ underH $\mathrm{C}_{0}$ where $\rho=\frac{\gamma_{1}}{\gamma_{0}}=1$. So the GLRT statistic is defined as

$$
Z_{l r t}=\frac{\sup \left\{L\left(\theta\left(\tilde{\gamma}_{1}=\tilde{\gamma}_{0}, \tilde{\phi}, \rho=1\right) \mid x_{0}, x_{1}\right): \theta\left(\gamma_{0}, \gamma_{1}, \phi\right) \in \Theta_{0}\right\}}{\sup \left\{L\left(\theta\left(\hat{\gamma}_{0}, \hat{\gamma}_{1}, \hat{\phi}\right) \mid x_{0}, x_{1}\right): \theta\left(\gamma_{0}, \gamma_{1}, \phi\right) \in \Theta\right\}}=\frac{\left.\left(\begin{array}{c}
\frac{n}{\tilde{\phi}}+x_{0}-1 \\
x_{0}
\end{array}\right)\left(\frac{\tilde{u}_{0} \tilde{\phi}}{\tilde{u}_{0} \tilde{\phi}+1}\right)^{x_{0}}\left(\frac{1}{u_{0} \tilde{\phi}+1}\right)^{\frac{n}{\tilde{\phi}} \times\left(\frac{n}{\tilde{\phi}}+x_{1}-1\right.} x_{1}\right)\left(\frac{w \tilde{u}_{0} \tilde{\phi}}{w \tilde{u}_{0} \tilde{\phi}+1}\right)^{x_{1}}\left(\frac{1}{w \tilde{u}_{0} \tilde{\phi}+1}\right)^{\frac{n}{\tilde{\phi}}}}{\left(\begin{array}{c}
\frac{n}{\hat{\phi}}+x_{0}-1 \\
x_{0}
\end{array}\right)\left(\frac{x_{0} \hat{\phi} / n}{x_{0} \hat{\phi} / n+1}\right)^{x_{0}}\left(\frac{1}{\frac{x_{0} \hat{\phi}}{n}+1}\right)^{\frac{n}{\tilde{\phi}}} \times\left(\begin{array}{c}
\frac{n}{\hat{\phi}}+x_{1}-1 \\
x_{1}
\end{array}\right)\left(\frac{x_{1} \hat{\phi} / n}{x_{1} \hat{\phi} / n+1}\right)^{x_{1}}\left(\frac{1}{\frac{x_{1} \hat{\phi}}{n}+1}\right)^{\frac{n}{\tilde{\phi}}}} .
$$


Citation: Li X, Cooper NGF, Shyr Y, Wu D, Rouchka EC, et al. (2017) Inference and Sample Size Calculations Based on Statistical Tests in a Negative Binomial Distribution for Differential Gene Expression in RNA-seq Data. J Biom Biostat 8: 332. doi:10.4172/2155-6180.1000332

Page 5 of 12

Since $T=-2 \ln Z_{l r t}=-2\left[\ln L\left(\tilde{\gamma}_{1}=\tilde{\gamma}_{0}, \tilde{\phi}, \rho=1\right)-\ln L\left(\hat{\gamma}_{0}, \hat{\gamma}_{1}, \hat{\phi}\right)\right]$ approximately follows a $\chi_{1}^{2}$ distribution, the p-value is approximately:

p.value $\left(x_{0}, x_{1}\right)=1-\chi_{1}^{2}(T)$.

The p-value in equation (16) is further adjusted by the FDR correction when multiple genes are used for the data analysis. Combining these together, the parameter estimates based on the MLEs from two assumptions and the following test statistics are summarized in Table 1.

\section{Sample size calculation for a single gene}

In order to calculate the sample size, a power function needs to be constructed. The power of a test is the probability that the null hypothesis is rejected when the alternative hypothesis is true. We derived the sample size under the specified power 1- $\beta$ and the significance level $\alpha$ with an assumption of a balanced design experiment between conditions (i.e., $\mathrm{n}_{0}=\mathrm{n}_{1}=\mathrm{n}$ ) and one-sided statistical test under $\mathrm{H}_{1}: \frac{\gamma_{1}}{\gamma_{0}}=\rho>1$.

Derivation of sample size based on the Wald test statistics: The details of derivation for each formula per test statistic are described in the Appendix: Derivation of sample size calculation in Supplementary Information. Briefly, given the parameters $\left(u_{0}\right.$ and $\left.\phi\right)$, the sample size formula based on the Wald test statistics $\left(\mathrm{Z}_{\mathrm{w} 1}, \mathrm{Z}_{\mathrm{lw1}}, \mathrm{Z}_{\mathrm{w} 2}\right.$ and $\left.\mathrm{Z}_{\mathrm{lw} 2}\right)$ are defined as

$$
\begin{aligned}
& n_{w 1}=\frac{\left(1+\rho / w+\hat{\phi} \rho^{2} u_{0}+\hat{\phi} u_{0}\right)\left(z_{1-\alpha}+z_{1-\beta}\right)^{2}}{u_{0}(\rho-1)^{2}} \\
& n_{l w 1}=\frac{\left(1+\frac{1}{\rho w}+2 \hat{\phi} u_{0}\right)\left(z_{1-\alpha}+z_{1-\beta}\right)^{2}}{u_{0}(\ln \rho)^{2}} ; \\
& n_{w 2}=\frac{\left(1+\rho / w+\tilde{\phi} \rho^{2} u_{0}+\tilde{\phi} u_{0}\right)\left(z_{1-\alpha} \sqrt{\frac{\tilde{u}_{0}(1 / w+1+2 \tilde{\phi} \tilde{u})}{u_{0}\left(\rho / w+1+\tilde{\phi} \rho^{2} u_{0}+\tilde{\phi} u_{0}\right)}}+z_{1-\beta}\right)^{2}}{u_{0}(\rho-1)^{2}} ; \\
& \text { and } n_{l w 2}=\frac{\left(\frac{1}{w \rho}+1+2 u_{0} \tilde{\phi}\right)\left(z_{1-\alpha} \sqrt{\frac{u_{0}\left(\frac{1}{w}+1+2 \tilde{u}_{0} \tilde{\phi}\right)}{\tilde{u}_{0}\left(\frac{1}{w \rho}+1+2 u_{0} \tilde{\phi}\right)}}+z_{1-\beta}\right)^{2}}{u_{0}(\ln \rho)^{2}}
\end{aligned}
$$

where $\tilde{u}_{0}$ is estimated from CMLE under $\mathrm{H}_{0}$ in equation (9) and $u_{0}$ is the mean read counts under $\mathrm{H}_{1}$ or the assumed true mean read counts in the control condition. Under the alternative hypothesis $H_{1}: \frac{\gamma_{1}}{\gamma_{0}}=\rho<1$, equations (17-20) are also true.

Derivation of sample size based on the likelihood ratio test (LRT) statistic: For the LRT with a negative binomial distribution, it is difficult to derive a closed-form expression of the power function. We used the method [19] to calculate the power of the LRT given a p-value from the equation (16) under LRT. This method originally borrowed a concept from this study [27] to calculate the power. Given a p-value based on the observed joint probability $P\left(X_{0}=x_{0}, X_{1}=x_{1}\right)$, the power under the assumption can be expressed as

$$
\operatorname{Pr}\left(n, \rho, u_{0}, \Phi, w, \alpha\right)=\sum_{x_{0}=0}^{\infty} \sum_{x_{1}=0}^{\infty} f\left(n w \rho u_{0}, \frac{\Phi}{n}\right) f\left(n u_{0}, \frac{\Phi}{n}\right) I\left(P\left(X_{0}=x_{0}, X_{1}=x_{1}\right)<\alpha\right),
$$

where $X_{0}$ and $X_{1}$ are independent, $f(u, \phi)$ is the probability mass function of the negative binomial distribution with mean $u$ and dispersion $\phi$, $\alpha$ is the level of significance, and $I(\cdot)$ is the indicator function of p-value. Thus, given a nominal power 1- $\beta$, the power of the test can be represented as the function of the sample size $n$ in the form of:

\begin{tabular}{|c|c|c|c|}
\hline Statistic tests & Maximum Likelihood estimates (MLE) & Statistical test & Log Transformed test \\
\hline \multirow[t]{2}{*}{ Wald test } & MLE under unrestricted parameter space & $Z_{w 1}$ & $Z_{l w 1}$ \\
\hline & Conditional MLE (CMLE) under $\mathrm{H}_{0}: \mathrm{Y}_{0}=\mathrm{Y}_{1}$ & $Z_{w 2}$ & $Z_{\text {lw2 }}$ \\
\hline Generalized Likelihood ratio test & CMLE/MLE & $Z_{l l t}$ & \\
\hline
\end{tabular}

$$
1-\beta=\operatorname{Pr}\left(n, \rho, u_{0}, \phi, w, \alpha\right) \text {. }
$$

Therefore, the required sample size $n$ to attain the nominal power $1-\beta$ at a significance level $\alpha$ can then be computed by solving equation (22) through a numerical approach with respect to $n$.

$\mathrm{V}_{0}$ and $\mathrm{Y}_{1}$ are true gene expression between two conditions.

Table 1: Statistical tests are used for deriving sample size calculations. The parameters are estimated using MLEs and CMLE methods. 
Citation: Li X, Cooper NGF, Shyr Y, Wu D, Rouchka EC, et al. (2017) Inference and Sample Size Calculations Based on Statistical Tests in a Negative Binomial Distribution for Differential Gene Expression in RNA-seq Data. J Biom Biostat 8: 332. doi:10.4172/2155-6180.1000332

\section{Sample size calculation with controlling FDR for testing multiple genes}

In an RNA-seq experiment, thousands of genes need to be tested simultaneously for DEGs between conditions. In this case, the sample size calculation for a single gene derived above needs to be further adjusted due to the multiple testing problems. In this section we derive sample size calculations by incorporating FDR controlling based on the statistical tests described in the previous sections. The details of controlling FDR have been given in the study [19]. Briefly, FDR $(f)$ is defined as

$$
f=\frac{m_{0} \alpha}{m_{0} \alpha+t_{1}},
$$

where $m_{0}$ is the number of true null hypotheses, $t_{1}=E\left(M_{1}\right)$ is the expected number of true rejections, $M_{0}$ is the number of false discoveries, $M$ is the total number of genes declared significant, $M_{1}=\mathrm{M}-M_{0}$ and $f$ is the control FDR at a specified level.

By solving equation (23) with respect to $\alpha$, the marginal type I error level $\alpha^{*}$ for the expected number of true rejections $\mathrm{t}_{1}$ at a given FDR $(f)$ is

$$
\alpha^{*}=\frac{t_{1} f}{m_{0}(1-f)} \text {. }
$$

Replacing $\alpha$ with $\alpha^{*}$ in equation (24) in equations (17-20), the corresponding sample size calculation formulas corrected by FDR at level $f$ are, respectively,

$$
\begin{aligned}
& n_{w 1}=\frac{\left(1+\rho / w+\hat{\phi} \rho^{2} u_{0}+\hat{\phi} u_{0}\right)\left(z_{1-\alpha^{*} / 2}+z_{1-\beta}\right)^{2}}{u_{0}(\rho-1)^{2}}, \\
& n_{l w 1}=\frac{\left(1+\frac{1}{\rho w}+2 \hat{\phi} u_{0}\right)\left(z_{1-\alpha^{*}}+z_{1-\beta}\right)^{2}}{u_{0}(\ln \rho)^{2}} \text {, } \\
& =\frac{\left(1+\rho / w+\tilde{\phi} \rho^{2} u_{0}+\tilde{\phi} u_{0}\right)\left(z_{1-\alpha^{*}} \sqrt{\frac{\tilde{u}_{0}\left(1 / w+1+2 \tilde{\phi} \tilde{u}_{0}\right)}{u_{0}\left(\rho / w+1+\tilde{\phi} \rho^{2} u_{0}+\tilde{\phi} u_{0}\right)}}+z_{1-\beta}\right)^{2}}{u_{0}(\rho-1)^{2}}, \\
& \frac{\left(\frac{1}{w \rho}+1+2 u_{0} \tilde{\phi}\right)\left(z_{1-\alpha^{*}} \sqrt{\frac{u_{0}\left(\frac{1}{w}+1+2 \tilde{u}_{0} \tilde{\phi}\right)}{\tilde{u}_{0}\left(\frac{1}{w \rho}+1+2 u_{0} \tilde{\phi}\right)}}+z_{1-\beta}\right)^{2}}{u_{0}(\ln \rho)^{2}} .
\end{aligned}
$$

Similarly, replacing $\alpha$ with $\alpha^{*}$ for the LRT statistic in equation (22), we obtain the function with respect to $n$ as

$$
1-\beta=\operatorname{Pr}\left(n, \rho, u_{0}, \phi, w, \alpha^{*}\right) \text {. }
$$

Thus, by solving (29) via a numerical approach, the sample size for controlling FDR at level $f$ can be obtained.

\section{Simulation Studies and Comparison of Results}

The proposed sample size formulas are derived from the likelihood function based on large sample theory with an approximate normal distribution. The simulation studies include two parts. In the first part, we calculated sample size based on testing single gene from the different formulas. In the second part, we calculated sample size based on testing the multiple genes using FDR adjusted significance $\alpha^{*}$ level. The parameter settings in our simulation studies are based on empirical data sets. A comparison of the simulated power for the sample sizes using different methods was performed.

\section{Sample size calculations and power estimation based on testing a single gene}

The purpose of this study is to compare the performance of sample size calculations with the estimated power from our formula with the method based on the exact test in the public study [19]. We set the following inputs based on a single gene. Let the type I error rate $\alpha=0.05$, the power $1-\beta=0.8$, the ratio of total size factors between two condition $\mathrm{w}=1$ and 1.2 , the mean counts of gene $g$ in control condition $u_{0}=1,5$ or 10 , the dispersion $\phi=0.1,0.5$ or 1 , and the fold changes $\rho=1.5,2,3$ or 4 . Since the read depth across samples in RNA-seq data is usually close to each other, we choose $\mathrm{w}=1.2$ instead of $\mathrm{w}=2$ [19]. For each combination of these designed settings, at first, we used our derived formulas in equations (17-20 and 22) to calculate the required sample size, respectively. Then, we calculated the sample size based on the exact test computed using the $\mathrm{R}$ codes with the same input settings. Moreover, for each designed setting, we generated 5000 simulations from independent negative binomial distributions based on the calculated sample size $n$ given the dispersion $\phi$ with different mean counts. For the control condition ( $i=0$ ), we used $\mathrm{R}$ to generate random samples given the mean $u_{0}$ and $\phi$. For the treatment condition $(\mathrm{i}=1)$, we generated random samples given mean $u_{1}=w \rho u_{0}$ and $\phi$. The test statistics in equations (10-13 and 14-16) were applied to each simulation sample and the empirical power was obtained as the proportion of simulation samples for which $H_{0}$ is rejected with the nominal type I error $\alpha=0.05$. The results are shown in which reports the estimated sample 
Citation: Li X, Cooper NGF, Shyr Y, Wu D, Rouchka EC, et al. (2017) Inference and Sample Size Calculations Based on Statistical Tests in a Negative Binomial Distribution for Differential Gene Expression in RNA-seq Data. J Biom Biostat 8: 332. doi:10.4172/2155-6180.1000332

size with associated empirical power given in parentheses under the case $\mathrm{w}=1$ and 1.2 .

\section{Sample size calculation based on testing multiple genes via FDR-controlling method}

In this study, we evaluated the performance of the sample size methods based on testing the multiple genes via FDR-controlling method rather than the type I error $\alpha$, which is widely used in the RNA-seq analysis. We set $\mathrm{m}=10,000, \mathrm{~m}_{1}=100, \mathrm{~m}_{0}=\mathrm{m}-\mathrm{m}_{1}$ and want to detect the expected number of true DEG $\mathrm{t}_{1}=80$ and the actual power corresponding to the nominal power of $1-\beta=80 \%$. We also set $\mathrm{u}_{\mathrm{og}}=1,5$ or $10, \rho_{\mathrm{g}}=1.5,2,3$ or 4 and $\phi_{\mathrm{g}}=0.1,0.5$ or 1.0. With these settings, the new $\alpha^{*}=4.25 \times 10^{-4}$ was obtained from the equation (24) at a desired FDR $(f=0.05)$. Then, we calculated the sample size by substituting $\alpha^{*}$ and power into the equations (25-29) and the published method using the exact test. For each designed setting, we also conducted 5000 simulations from an independent negative binomial distribution. The number of true DEGs was counted using $p$-values $\leq \alpha^{*}$ which is much smaller than the nominal type I error rate 0.05 . The empirical power was obtained as the proportion of simulation samples for which $H_{0}$ is rejected with the nominal type I error $\alpha^{*}=4.25 \times 10^{-4}$. The expected number of true DEGs under $\alpha^{*}$ can be estimated via the multiplication of the estimated power with the total number of true DEGs. The results in Table 2 report the estimated sample size with the empirical power given in parentheses under the cases $\mathrm{w}=1$ and 1.2 .

Given the sample size obtained from $\mathrm{n}_{\mathrm{lw} 2}$, an empirical power for other methods is also computed from 5,000 simulations (Table 3 ). In order to compare the performance of these methods the paired Wilcoxon signed-rank test is used to test the simulated power in Table 3 for the statistical significance. The results are in Table 4.

\section{Identify the pattern of the sample sizes changing with different values of $u_{0}, \phi$ and $\rho$ for different methods at $\alpha$ and $\alpha^{*}$ levels}

First, we identified patterns of sample size changes with different values of $u_{0}$, $\phi$ and $\rho$ for different methods. Although Table 2 shows similar pattern of sample sizes obtained from the six methods, we found that the required sample sizes $n_{l w 1}$ and $n_{l w 2}$ derived from the log-transformed Wald tests are the smallest compared with the other methods.

Figure 1 illustrates $n_{l w 2}$ varying with the values of $u_{0}$ when other parameters, such as $\rho \in(1.5,2,3,4), \phi \in(.1, .5,1)$ and $w \in(1,1.2)$ are fixed Given the nominal power $(1-\beta=0.8)$ and $\alpha=0.05$ or FDR= $=0.05$, as expected, $n_{\text {lw2 }}$ decreases as $u_{0}$ increases under a fixed $\rho$ and $\phi$. This indicates that for a lowly expressed gene, a larger sample size is required to achieve a detection power of DEGs between two conditions. For a fixed $u_{0}$ and $\rho, n_{l w 2}$ increases as $\phi$ increases (Figure 1). This is also expected because a larger $\phi$ indicates higher variation of the genes across conditions. Furthermore, for a fixed $\phi$ and $u_{0}, n_{l w 2}$ decreases as $\rho$ increases. This result indicates a smaller $n$ is required for a larger difference of mean read counts between two conditions or vice versa. For the same setting of parameters, we found the $n_{l w 2}$ in Figure $1 \mathrm{~A}$ and $1 \mathrm{C}$ with an equal size factor $(\mathrm{w}=1)$ across conditions is slightly larger than the unequal size factor in Figure $1 \mathrm{~B}(\mathrm{w}=1)$. Under the same settings, as expected, the sample sizes in Table 2 with FDR=0.05 are larger than those with $\alpha=0.05$, indicating that a larger $n$ is required for detecting DEGs while testing thousands of genes simultaneously (Figure $1 \mathrm{C}$ and $1 \mathrm{D})$ compared with testing a single gene (Figure1 $\mathrm{A}$ and $1 \mathrm{~B})$.

Comparison of the sample calculations from different methods based on testing a single genes in multiple Table S1 genes in Table 2

Next we compared the sample $\operatorname{siz}^{\wedge}(n)$ estimated from our five derived methods $\left(n_{w 1}, n_{w 2}, n_{l w 1}, n_{l w 2}, n_{l r}\right)$ and the public method ( $\left.n_{\text {exact }}\right)$ [19]. Figure 2 illustrates that $n$ decreases as ${ }^{u_{0}}$ increases for all methods given $w=1$, power $=0.8, \operatorname{FDR}=0.05, \phi \in(0.1,0.5,1)$ and $\rho \in(1.5,2)$. Given $\rho=1.5$, the sample sizes from all methods are getting close to each other when $\phi$ is 0.1 for small biological variation (Figure $2 \mathrm{~A}-2 \mathrm{C}$ ). As $\phi$ increases, the difference between the sample sizes for all methods becomes much larger. Similar patterns were observed given $\rho=2$ (Figure $2 \mathrm{D}-2 \mathrm{~F}$ ). With regards to the $n$, we noticed that the sample sizes calculated from $n_{l w 1}$ and $n_{l w 2}$ methods (red in Figure 2) are the smallest while maintaining the nominal power close to or above $80 \%$. Among the other four methods, no one performed better than others in all scenarios.

In addition, the empirical power in parentheses of Table S1 and Table 2 was calculated from simulations with the size of 5,000 corresponding to the estimated $\mathrm{n}$ for all methods. The results show almost all of the methods are close to or higher than the desired power. Although the sample sizes calculated using $n_{l w 1}$ and $n_{l w 2}$ are the smallest, we cannot arbitrarily conclude that these two methods are the best because the corresponding empirical powers are varied corresponding to the sample sizes for each method (Table 2).

For a better comparison with the same settings and a fixed and small $\mathrm{n}$ estimated from the log-transformed Wald method $\left(n_{l w 2}\right)$, we observed that $n_{l w 1}$ and $n_{l w 2}$ consistently achieve a better power close to the nominal power $80 \%$ or higher in all scenarios compared to other methods (Table 3). We also observed that $n_{l t r}$ and $n_{\text {exact }}$ perform similarly and both of them achieve a higher power than $n_{w 1}$ when the fold change is great than 2. However, $n_{w 1}$ achieves a better power than $n_{l r t}$ and $n_{\text {exact }}$ when the fold change is at $\rho \leq 2$ (Table 3 ). Table 4 from a paired Wilcoxon ranked test indicates that the empirical power from $n_{l w 2}$ is statistically significant from that achieved using other methods. Table 4 also shows that $n_{l w 1}$ performed significantly better than others four methods. Among these four methods, no one performed better than the others in all scenarios.

\section{Application}

Sample size calculation based on RNA-seq data in human breast cancer: To identify DEGs between two conditions, we explored a real human breast cancer dataset to calculate the sample size. Forty Estrogen receptor positive (ER + ) and HER2 negative breast cancer primary tumors and 29 uninvolved breast tissue sample that were adjacent to ER+ primary tumors in .fastq format were downloaded from NCBI GEO (series ID GSE58135). The raw sequencing files were mapped to the human hg19 reference genome using tophat2 (v2.0.13) with bowtie version (2.2.3.0). The mapped counts for each gene per sample were then extracted using HTSeq-scripts-count (version 2.7). There are a total of 57,773 genes extracted. After filtering genes with the mean read counts less than one in two groups, 35,112 genes were left. These samples were loaded into edgeR to estimate common dispersion and size factors. With the aid of edgeR, the normalization factors called size factors are estimated using the "RLE" scaling factor 
Citation: Li X, Cooper NGF, Shyr Y, Wu D, Rouchka EC, et al. (2017) Inference and Sample Size Calculations Based on Statistical Tests in a Negative Binomial Distribution for Differential Gene Expression in RNA-seq Data. J Biom Biostat 8: 332. doi:10.4172/2155-6180.1000332

Page 8 of 12

\begin{tabular}{|c|c|c|c|c|c|c|c|c|c|}
\hline w & $\rho$ & 中 & $u_{0}$ & $n_{w 1}$ & $n_{w 2}$ & $n_{l w 1}$ & $n_{1 w 2}$ & $n_{\text {Irt }}$ & $n_{\text {exact }}$ \\
\hline \multirow[t]{36}{*}{1} & \multirow[t]{9}{*}{1.5} & \multirow[t]{3}{*}{.1} & 1 & $197(0.80)$ & $196(0.81)$ & $198(0.81)$ & $192(0.80)$ & $221(0.82)$ & $218(0.80)$ \\
\hline & & & 5 & $58(0.81)$ & $57(0.81)$ & $57(0.81)$ & $55(0.79)$ & $65(0.84)$ & $63(0.81)$ \\
\hline & & & 10 & $40(0.81)$ & $39(0.80)$ & $39(0.80)$ & $38(0.79)$ & $45(0.84)$ & $43(0.80)$ \\
\hline & & \multirow[t]{3}{*}{0.5} & 1 & $288(0.81)$ & $284(0.82)$ & $283(0.81)$ & $277(0.80)$ & $321(0.83)$ & $311(0.80)$ \\
\hline & & & 5 & $148(0.82)$ & $145(0.81)$ & $142(0.80)$ & $140(0.80)$ & $162(0.83)$ & $156(0.81)$ \\
\hline & & & 10 & $131(0.82)$ & $127(0.80)$ & $124(0.81)$ & $123(0.80)$ & $142(0.83)$ & $137(0.81)$ \\
\hline & & \multirow[t]{3}{*}{1} & 1 & $401(0.81)$ & $394(0.81)$ & $389(0.81)$ & $384(0.80)$ & $441(0.82)$ & $429(0.80)$ \\
\hline & & & 5 & $262(0.82)$ & $255(0.81)$ & $248(0.80)$ & $247(0.80)$ & $284(0.83)$ & $273(0.80)$ \\
\hline & & & 10 & $244(0.83)$ & $237(0.82)$ & $230(0.81)$ & $229(0.81)$ & $264(0.84)$ & $254(0.81)$ \\
\hline & \multirow[t]{9}{*}{2} & \multirow[t]{3}{*}{.1} & 1 & $61(0.82)$ & $60(0.81)$ & $62(0.83)$ & $57(0.80)$ & $67(0.83)$ & $67(0.81)$ \\
\hline & & & 5 & $19(0.83)$ & $18(0.82)$ & $18(0.82)$ & $17(0.80)$ & $21(0.85)$ & $20(0.82)$ \\
\hline & & & 10 & $14(0.84)$ & $13(0.82)$ & $13(0.82)$ & $12(0.80)$ & $15(0.86)$ & $14(0.81)$ \\
\hline & & \multirow[t]{3}{*}{0.5} & 1 & $96(0.83)$ & $92(0.82)$ & $91(0.81)$ & $86(0.80)$ & $101(0.83)$ & $99(0.80)$ \\
\hline & & & 5 & $54(0.85)$ & $51(0.84)$ & $47(0.81)$ & $46(0.80)$ & $54(0.84)$ & $53(0.82)$ \\
\hline & & & 10 & $49(0.84)$ & $45(0.82)$ & $42(0.80)$ & $41(0.79)$ & $48(0.82)$ & $47(0.82)$ \\
\hline & & \multirow[t]{3}{*}{1} & 1 & $140(0.83)$ & $133(0.81)$ & $127(0.80)$ & $122(0.80)$ & $143(0.82)$ & $140(0.80)$ \\
\hline & & & 5 & $98(0.85)$ & $91(0.84)$ & $84(0.80)$ & $83(0.80)$ & $96(0.83)$ & $93(0.80)$ \\
\hline & & & 10 & $92(0.84)$ & $85(0.83)$ & $78(0.81)$ & $78(0.81)$ & $90(0.83)$ & $87(0.81)$ \\
\hline & \multirow[t]{9}{*}{3} & \multirow[t]{3}{*}{0.1} & 1 & $22(0.84)$ & $21(0.83)$ & $22(0.84)$ & $18(0.80)$ & $23(0.85)$ & $23(0.83)$ \\
\hline & & & 5 & $8(0.88)$ & $7(0.83)$ & $7(0.84)$ & $6(0.79)$ & $8(0.88)$ & $8(0.87)$ \\
\hline & & & 10 & $6(0.87)$ & $5(0.80)$ & $5(0.82)$ & $4(0.74)$ & $6(0.89)$ & $6(0.88)$ \\
\hline & & \multirow[t]{3}{*}{0.5} & 1 & $39(0.86)$ & $36(0.85)$ & $34(0.83)$ & $30(0.80)$ & $36(0.82)$ & $37(0.82)$ \\
\hline & & & 5 & $25(0.89)$ & $22(0.87)$ & $18(0.80)$ & $18(0.82)$ & $21(0.83)$ & $21(0.82)$ \\
\hline & & & 10 & $24(0.91)$ & $20(0.87)$ & $16(0.79)$ & $16(0.80)$ & $19(0.83)$ & $19(0.82)$ \\
\hline & & 1 & 1 & $61(0.87)$ & $54(0.85)$ & $48(0.82)$ & $44(0.79)$ & $54(0.83)$ & $53(0.81)$ \\
\hline & & & 5 & $47(0.91)$ & $40(0.87)$ & $33(0.81)$ & $32(0.80)$ & $38(0.83)$ & $37(0.81)$ \\
\hline & & & 10 & $45(0.91)$ & $38(0.88)$ & $31(0.81)$ & $30(0.80)$ & $36(0.83)$ & $35(0.81)$ \\
\hline & 4 & 0.1 & 1 & $13(0.85)$ & $12(0.83)$ & $13(0.85)$ & $10(0.81)$ & $13(0.85)$ & $13(0.82)$ \\
\hline & & & 5 & $5(0.88)$ & $5(0.92)$ & $4(0.82)$ & $3(0.72)$ & $5(0.91)$ & $5(0.89)$ \\
\hline & & & 10 & $4(0.88)$ & $4(0.93)$ & $3(0.83)$ & $3(0.87)$ & $4(0.92)$ & $4(0.91)$ \\
\hline & & 0.5 & 1 & $26(0.89)$ & $23(0.88)$ & $20(0.82)$ & $17(0.79)$ & $22(0.84)$ & $22(0.82)$ \\
\hline & & & 5 & $18(0.93)$ & $15(0.91)$ & $11(0.79)$ & $11(0.81)$ & $13(0.83)$ & $13(0.82)$ \\
\hline & & & 10 & $17(0.94)$ & $14(0.91)$ & $10(0.79)$ & $10(0.81)$ & $12(0.82)$ & $12(0.82)$ \\
\hline & & 1 & 1 & $43(0.93)$ & $36(0.89)$ & $30(0.82)$ & $26(0.79)$ & $33(0.84)$ & $33(0.83)$ \\
\hline & & & 5 & $35(0.95)$ & $28(0.91)$ & $20(0.79)$ & $20(0.80)$ & $24(0.84)$ & $24(0.83)$ \\
\hline & & & 10 & $34(0.95)$ & $27(0.92)$ & $19(0.80)$ & $19(0.81)$ & $23(0.84)$ & $23(0.83)$ \\
\hline 1.2 & 1.5 & 0.1 & 1 & $180(0.80)$ & $184(0.80)$ & $186(0.81)$ & $177(0.80)$ & $205(0.88)$ & $202(0.80)$ \\
\hline & & & 5 & $54(0.80)$ & $55(0.81)$ & $54(0.80)$ & $52(0.79)$ & $62(0.88)$ & $60(0.80)$ \\
\hline & & & 10 & $38(0.80)$ & $38(0.80)$ & $38(0.81)$ & $37(0.80)$ & $43(0.88)$ & $42(0.82)$ \\
\hline & & 0.5 & 1 & $270(0.80)$ & $273(0.80)$ & $271(0.80)$ & $262(0.80)$ & $306(0.88)$ & $296(0.80)$ \\
\hline & & & 5 & $145(0.82)$ & $143(0.81)$ & $139(0.81)$ & $138(0.81)$ & $159(0.88)$ & $153(0.81)$ \\
\hline & & & 10 & $129(0.82)$ & $126(0.82)$ & $123(0.82)$ & $122(0.81)$ & $140(0.88)$ & $135(0.82)$ \\
\hline & & 1 & 1 & $384(0.81)$ & $383(0.81)$ & $377(0.81)$ & $369(0.80)$ & $427(0.88)$ & $413(0.80)$ \\
\hline & & & 5 & $258(0.82)$ & $253(0.81)$ & $245(0.80)$ & $244(0.80)$ & $279(0.88)$ & $269(0.80)$ \\
\hline & & & 10 & $243(0.83)$ & $236(0.82)$ & $229(0.80)$ & $228(0.80)$ & $260(0.88)$ & $251(0.81)$ \\
\hline & 2 & 0.1 & 1 & $55(0.80)$ & $57(0.81)$ & $59(0.83)$ & $52(0.80)$ & $62(0.87)$ & $62(0.80)$ \\
\hline & & & 5 & $18(0.83)$ & $18(0.83)$ & $18(0.83)$ & $16(0.80)$ & $20(0.89)$ & $19(0.81)$ \\
\hline & & & 10 & $13(0.83)$ & $13(0.83)$ & $12(0.79)$ & $12(0.81)$ & $14(0.89)$ & $14(0.84)$ \\
\hline & & 0.5 & 1 & $90(0.82)$ & $90(0.82)$ & $88(0.81)$ & $82(0.81)$ & $97(0.88)$ & $95(0.80)$ \\
\hline & & & 5 & $53(0.85)$ & $50(0.83)$ & $47(0.81)$ & $45(0.80)$ & $53(0.88)$ & $52(0.82)$ \\
\hline & & & 10 & $48(0.84)$ & $45(0.83)$ & $41(0.79)$ & $41(0.80)$ & $48(0.88)$ & $46(0.80)$ \\
\hline & & 1 & 1 & $134(0.82)$ & $130(0.81)$ & $124(0.80)$ & $118(0.80)$ & $139(0.87)$ & $136(0.80)$ \\
\hline & & & 5 & $97(0.84)$ & $90(0.83)$ & $83(0.80)$ & $82(0.79)$ & $95(0.87)$ & $92(0.81)$ \\
\hline & & & 10 & $92(0.85)$ & $85(0.84)$ & $78(0.81)$ & $77(0.80)$ & $89(0.87)$ & $86(0.81)$ \\
\hline & 3 & 0.1 & 1 & $20(0.85)$ & $20(0.83)$ & $21(0.85)$ & $17(0.81)$ & $21(0.88)$ & $22(0.84)$ \\
\hline & & & 5 & $7(0.82)$ & $7(0.84)$ & $7(0.86)$ & $6(0.82)$ & $7(0.87)$ & $7(0.80)$ \\
\hline & & & 10 & $6(0.89)$ & $5(0.81)$ & $5(0.84)$ & $4(0.76)$ & $6(0.93)$ & $6(0.89)$ \\
\hline & & 0.5 & 1 & $37(0.86)$ & $35(84)$ & $33(0.82)$ & $29(0.81)$ & $35(0.87)$ & $35(0.81)$ \\
\hline & & & 5 & $25(0.90)$ & $22(0.88)$ & $18(0.80)$ & $17(0.79)$ & $21(0.89)$ & $21(0.83)$ \\
\hline & & & 10 & $23(0.90)$ & $20(0.88)$ & $16(0.79)$ & $16(0.80)$ & $19(0.88)$ & $19(0.82)$ \\
\hline & & 1 & 1 & $59(0.89)$ & $53(0.85)$ & $47(0.81)$ & $43(0.79)$ & $53(0.93)$ & $52(0.81)$ \\
\hline & & & 5 & $47(0.91)$ & $40(0.88)$ & $33(0.82)$ & $32(0.81)$ & $38(0.88)$ & $37(0.82)$ \\
\hline & & & 10 & $45(0.91)$ & $38(0.88)$ & $31(0.81)$ & $30(0.80)$ & $36(0.89)$ & $35(0.82)$ \\
\hline
\end{tabular}


Citation: Li X, Cooper NGF, Shyr Y, Wu D, Rouchka EC, et al. (2017) Inference and Sample Size Calculations Based on Statistical Tests in a Negative Binomial Distribution for Differential Gene Expression in RNA-seq Data. J Biom Biostat 8: 332. doi:10.4172/2155-6180.1000332

Page 9 of 12

\begin{tabular}{|c|c|c|c|c|c|c|c|c|}
\hline \multirow[t]{9}{*}{4} & \multirow[t]{3}{*}{0.1} & 1 & $12(0.87)$ & $12(0.86)$ & $13(0.88)$ & $9(0.90)$ & $12(0.89)$ & $12(0.81)$ \\
\hline & & 5 & $5(0.91)$ & $4(0.81)$ & $4(0.84)$ & $3(0.75)$ & $5(0.95)$ & $5(0.91)$ \\
\hline & & 10 & $4(0.90)$ & $4(0.94)$ & $3(0.83)$ & $3(0.87)$ & $4(0.95)$ & $4(0.92)$ \\
\hline & \multirow[t]{3}{*}{0.5} & 1 & $25(0.90)$ & $22(0.87)$ & $20(0.84)$ & $16(0.79)$ & $21(0.89)$ & $21(0.82)$ \\
\hline & & 5 & $18(0.94)$ & $15(0.91)$ & $11(0.80)$ & $11(0.82)$ & $13(0.88)$ & $13(0.82)$ \\
\hline & & 10 & $17(0.94)$ & $14(0.91)$ & $10(0.79)$ & $10(0.81)$ & $12(0.88)$ & $12(0.83)$ \\
\hline & \multirow[t]{3}{*}{1} & 1 & $41(0.92)$ & $35(0.88)$ & $29(0.81)$ & $26(0.81)$ & $32(0.88)$ & $32(0.82)$ \\
\hline & & 5 & $35(0.95)$ & $28(0.91)$ & $20(0.80)$ & $20(0.81)$ & $24(0.89)$ & $24(0.83)$ \\
\hline & & 10 & $34(0.95)$ & $27(0.92)$ & $19(0.80)$ & $19(0.80)$ & $23(0.89)$ & $22(0.81)$ \\
\hline
\end{tabular}

$n_{w 1}, n_{w 2}, n_{l w 1}$ and $n_{l w 2}$ are our methods and $n_{\text {exact }}$ is the public method.

Table 2: Simulated power for testing multiple genes with sample sizes. The sample sizes are calculated using six methods given a predefined FDR=0.05and $80 \%$ power.

\begin{tabular}{|c|c|c|c|c|c|c|c|c|c|}
\hline & $\rho$ & 中 & $u_{0}$ & $n_{l w 2}$ & $n_{\mid w 1}$ & $n_{w 1}$ & $n_{w 2}$ & $n_{\text {Irt }}$ & $n_{\text {exact }}$ \\
\hline \multirow[t]{36}{*}{$w=1$} & \multirow[t]{9}{*}{1.5} & \multirow[t]{3}{*}{0.1} & 1 & $192(0.80)$ & 0.79 & 0.79 & 0.79 & 0.74 & 0.72 \\
\hline & & & 5 & $55(0.79)$ & 0.78 & 0.78 & 0.78 & 0.73 & 0.72 \\
\hline & & & 10 & $38(0.79)$ & 0.78 & 0.77 & 0.78 & 0.73 & 0.72 \\
\hline & & \multirow[t]{3}{*}{0.5} & 1 & $277(0.80)$ & 0.79 & 0.79 & 0.78 & 0.73 & 0.72 \\
\hline & & & 5 & $140(0.80)$ & 0.79 & 0.78 & 0.78 & 0.74 & 0.73 \\
\hline & & & 10 & $123(0.80)$ & 0.80 & 0.78 & 0.80 & 0.75 & 0.74 \\
\hline & & \multirow[t]{3}{*}{1} & 1 & $384(0.80)$ & 0.80 & 0.78 & 0.78 & 0.74 & 0.74 \\
\hline & & & 5 & $247(0.80)$ & 0.80 & 0.78 & 0.79 & 0.74 & 0.73 \\
\hline & & & 10 & $229(0.81)$ & 0.81 & 0.79 & 0.80 & 0.75 & 0.74 \\
\hline & \multirow[t]{9}{*}{2} & \multirow[t]{3}{*}{0.1} & 1 & $57(0.80)$ & 0.77 & 0.77 & 0.78 & 0.73 & 0.70 \\
\hline & & & 5 & $17(0.80)$ & 0.78 & 0.76 & 0.78 & 0.73 & 0.71 \\
\hline & & & 10 & $12(0.80)$ & 0.78 & 0.74 & 0.77 & 0.72 & 0.71 \\
\hline & & \multirow[t]{3}{*}{0.5} & 1 & $86(0.80)$ & 0.78 & 0.76 & 0.77 & 0.73 & 0.71 \\
\hline & & & 5 & $46(0.80)$ & 0.79 & 0.74 & 0.77 & 0.73 & 0.72 \\
\hline & & & 10 & $41(0.79)$ & 0.78 & 0.72 & 0.76 & 0.72 & 0.72 \\
\hline & & \multirow[t]{3}{*}{1} & 1 & $122(0.80)$ & 0.78 & 0.74 & 0.77 & 0.73 & 0.71 \\
\hline & & & 5 & $83(0.80)$ & 0.79 & 0.73 & 0.77 & 0.73 & 0.72 \\
\hline & & & 10 & $78(0.81)$ & 0.81 & 0.74 & 0.78 & 0.75 & 0.74 \\
\hline & \multirow[t]{9}{*}{3} & \multirow[t]{3}{*}{0.1} & 1 & $18(0.80)$ & 0.70 & 0.71 & 0.71 & 0.70 & 0.64 \\
\hline & & & 5 & $6(0.79)$ & 0.74 & 0.66 & 0.73 & 0.69 & 0.66 \\
\hline & & & 10 & $4(0.74)$ & 0.70 & 0.53 & 0.65 & 0.63 & 0.61 \\
\hline & & \multirow[t]{3}{*}{0.5} & 1 & $30(0.80)$ & 0.74 & 0.66 & 0.73 & 0.70 & 0.66 \\
\hline & & & 5 & $18(0.82)$ & 0.80 & 0.63 & 0.75 & 0.74 & 0.72 \\
\hline & & & 10 & $16(0.80)$ & 0.79 & 0.59 & 0.73 & 0.72 & 0.71 \\
\hline & & \multirow[t]{3}{*}{1} & 1 & $44(0.79)$ & 0.76 & 0.62 & 0.72 & 0.70 & 0.67 \\
\hline & & & 5 & $32(0.80)$ & 0.79 & 0.57 & 0.73 & 0.72 & 0.71 \\
\hline & & & 10 & $30(0.80)$ & 0.79 & 0.55 & 0.72 & 0.72 & 0.71 \\
\hline & \multirow[t]{9}{*}{4} & \multirow[t]{3}{*}{0.1} & 1 & $10(0.81)$ & 0.65 & 0.62 & 0.71 & 0.67 & 0.62 \\
\hline & & & 5 & $3(0.72)$ & 0.62 & 0.39 & 0.58 & 0.58 & 0.53 \\
\hline & & & 10 & $3(0.87)$ & 0.81 & 0.62 & 0.78 & 0.78 & 0.76 \\
\hline & & \multirow[t]{3}{*}{0.5} & 1 & $17(0.79)$ & 0.71 & 0.52 & 0.67 & 0.67 & 0.63 \\
\hline & & & 5 & $11(0.81)$ & 0.79 & 0.41 & 0.69 & 0.71 & 0.70 \\
\hline & & & 10 & $10(0.81)$ & 0.79 & 0.33 & 0.68 & 0.71 & 0.70 \\
\hline & & \multirow[t]{3}{*}{1} & 1 & $26(0.79)$ & 0.74 & 0.44 & 0.66 & 0.67 & 0.64 \\
\hline & & & 5 & $20(0.80)$ & 0.79 & 0.34 & 0.68 & 0.71 & 0.69 \\
\hline & & & 10 & $19(0.81)$ & 0.80 & 0.30 & 0.68 & 0.72 & 0.71 \\
\hline
\end{tabular}

$n_{w 1}, n_{w 2}, n_{l w 1}$ and $n_{l w 2}$ are our methods and $n_{\text {exact }}$ is the public method.

Table 3: Simulated power for testing multiple genes given the sample size. The sample sizes are computed using $\boldsymbol{n}_{\mathrm{lw2}}$ method at a predefined FDR=0.05 and $80 \%$ power.

method [17] and the estimated ratio of total size factors of the samples in each condition $(w)$ is 1.1 . The common dispersion $\phi$ is estimated as 0.48 using the CMLE method [22].

We assumed the top 400 of 35,122 genes (1.1\%) are prognostic and have the largest fold changes. The minimum of average read counts among these genes in the control group served as pilot data was estimated as $u_{0}=1$ [16]. In addition, the sample sizes were estimated using $u_{0}=5,10$ and 20 . Suppose we want to set the nominal power to $80 \%$, which indicates we want to identify 320 or more of the prognostic genes. Under the control FDR at $f=0.10$ and $80 \%$ power, we can set $\mathrm{m}=35,112, \mathrm{~m}_{1}=400, m_{0}=m-m_{1}$ and $\mathrm{t}_{1}=320$. The parameters $\rho_{\mathrm{g}}(\mathrm{g}=1, \ldots, 35,112)$ were assumed to be unknown. Let the mean counts in the control condition $u_{0 g}=1,5,10$, or 20 and fold change $\rho_{\mathrm{g}}=1.5$ or 2,3 and 4 with common dispersion $\phi_{\mathrm{g}}=0.48$. With these settings, the new $\alpha^{*}=9.992 \times 10^{-4}$ was obtained from equation (24) at a desired $\operatorname{FDR}(f=0.10)$. Then, we calculated the sample size by substituting $\alpha^{*}$ and the power into equations (25-29) for each method (Table 5). 
Citation: Li X, Cooper NGF, Shyr Y, Wu D, Rouchka EC, et al. (2017) Inference and Sample Size Calculations Based on Statistical Tests in a Negative Binomial Distribution for Differential Gene Expression in RNA-seq Data. J Biom Biostat 8: 332. doi:10.4172/2155-6180.1000332

Page 10 of 12

\begin{tabular}{|l|l|l|l|l|l|}
\hline$n_{w 1}$ & $n_{l w 1}$ & $n_{w 2}$ & $n_{\text {exact }}$ & $n_{\text {Irt }}$ & \\
\hline$n_{l w 2}$ & $<0.0001^{*}$ & $<0.0001^{*}$ & $<0.0001^{*}$ & $<0.0001^{*}$ \\
\hline$n_{l w 1}$ & - & $<0.001^{*}$ & $<0.0001^{*}$ & $<0.0001^{*}$ \\
\hline$n_{w 2}$ & - & $<0.001^{*}$ & $<0.0001^{*}$ \\
\hline$n_{\text {exact }}$ & & & - & $<0.0001^{*}$ \\
\hline$n_{l t+}$ & & & & $<0.05^{*}$ \\
\hline
\end{tabular}

*Statistically significant.

Table 4: P-values are calculated using the paired Wilcoxon signed-rank statistical test of the power values in Table 3.

A. $w=1, F D R=0.05, \rho=1.5, \phi=0.1$

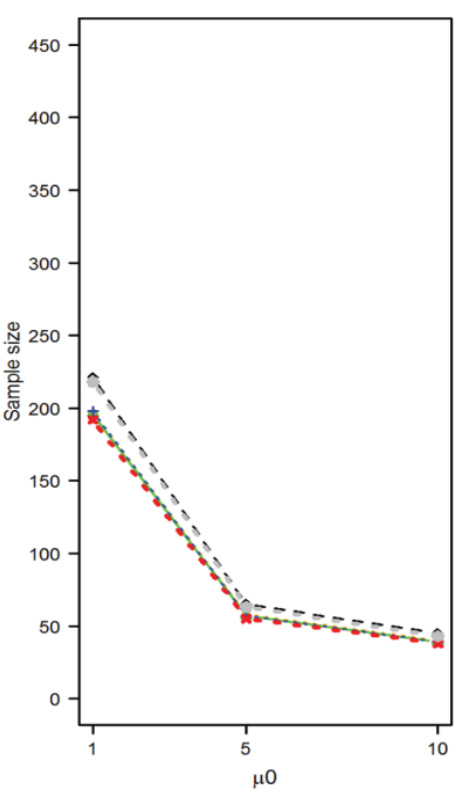

D. $\rho=2, \phi=0.1$

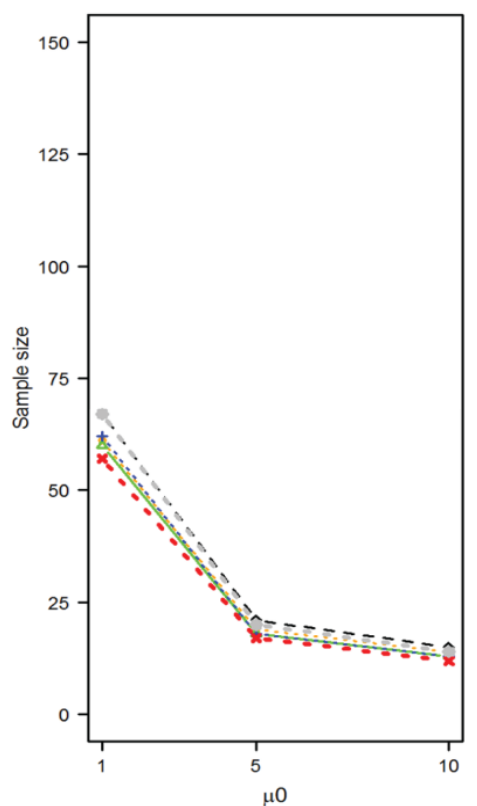

B. $\rho=1.5, \phi=0.5$

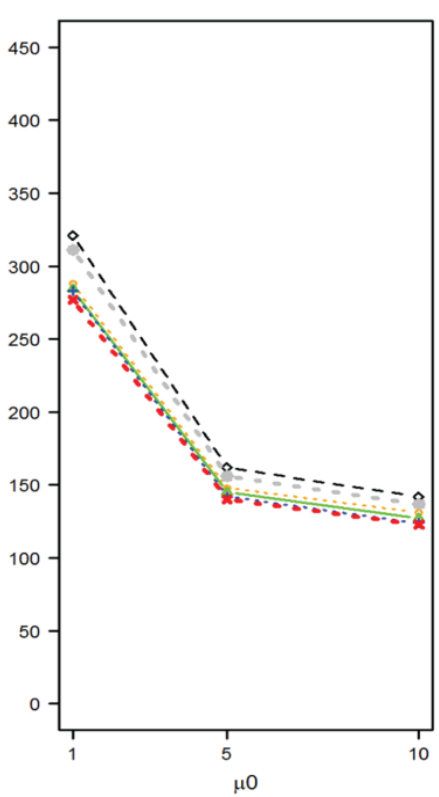

E. $\rho=2, \phi=0.5$

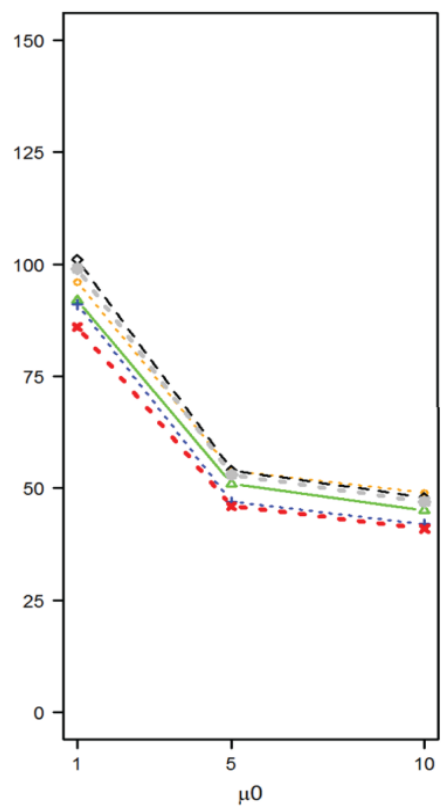

C. $\rho=1.5, \phi=1$

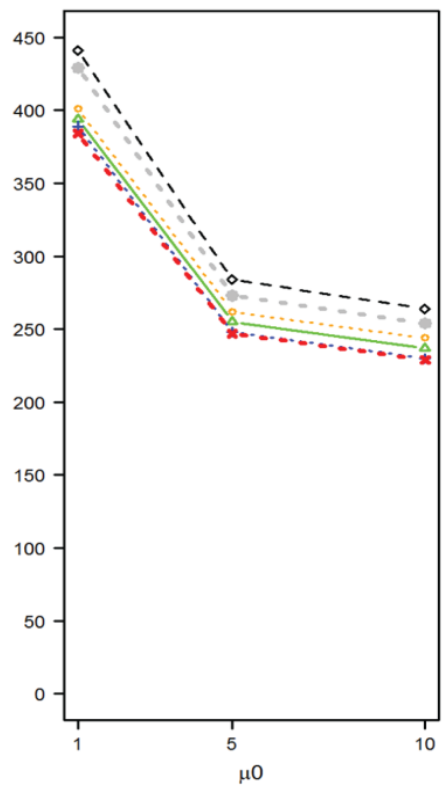

F. $\rho=2, \phi=1$

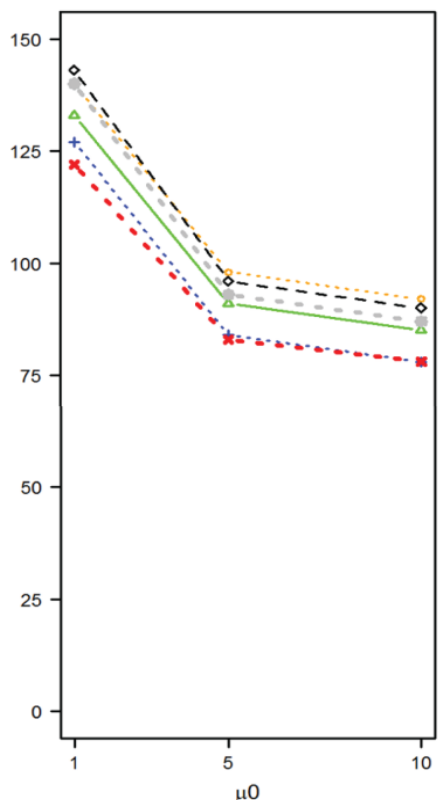

Figure 2: Sample size $(\mathrm{n})$ for testing multiple genes is required using the six methods $n_{w 1}, n_{w 2}, n_{l w 1}, n_{l w 2}, n_{l t r}$ and $n_{\text {exact }}$ ). $n$ varies with mean counts in control condition $\left(u_{0}\right)$ given the combination of fold change $(\rho)$ and dispersion $(\phi)$ at $w=1,80 \%$ power and 0.05 FDR. $n_{l w 1}$ and $n_{t w 2}$ methods in red and blue respectively require smaller $n$. Figures 2A and 2D illustrate samples sizes for all methods are close to each other when the dispersion $\phi$ is 0.1 . 
Citation: Li X, Cooper NGF, Shyr Y, Wu D, Rouchka EC, et al. (2017) Inference and Sample Size Calculations Based on Statistical Tests in a Negative Binomial Distribution for Differential Gene Expression in RNA-seq Data. J Biom Biostat 8: 332. doi:10.4172/2155-6180.1000332

Page 11 of 12

\begin{tabular}{|c|c|c|c|c|c|c|c|c|}
\hline & $\rho$ & $u_{0}$ & $n_{w 1}$ & $n_{w 2}$ & $n_{l w 1}$ & $n_{l w 2}$ & $n_{l r t}$ & $\boldsymbol{n}_{\text {exact }}$ \\
\hline \multirow{16}{*}{$\begin{array}{l}w=1.1 \\
\phi=.48\end{array}$} & \multirow[t]{4}{*}{1.5} & 1 & 242 & 241 & 240 & 234 & 275 & 267 \\
\hline & & 5 & 125 & 123 & 120 & 119 & 139 & 134 \\
\hline & & 10 & 111 & 108 & 105 & 104 & 122 & 117 \\
\hline & & 20 & 103 & 101 & 97 & 97 & 114 & 109 \\
\hline & \multirow[t]{4}{*}{2} & 1 & 80 & 79 & 77 & 73 & 87 & 86 \\
\hline & & 5 & 46 & 43 & 40 & 39 & 47 & 45 \\
\hline & & 10 & 41 & 39 & 35 & 35 & 42 & 40 \\
\hline & & 20 & 39 & 36 & 33 & 33 & 39 & 38 \\
\hline & \multirow[t]{4}{*}{3} & 1 & 33 & 31 & 29 & 25 & 32 & 32 \\
\hline & & 5 & 21 & 19 & 16 & 15 & 18 & 18 \\
\hline & & 10 & 20 & 17 & 14 & 14 & 17 & 16 \\
\hline & & 20 & 19 & 16 & 13 & 13 & 16 & 15 \\
\hline & \multirow[t]{4}{*}{4} & 1 & 22 & 19 & 18 & 15 & 19 & 19 \\
\hline & & 5 & 16 & 13 & 10 & 9 & 12 & 11 \\
\hline & & 10 & 15 & 12 & 9 & 8 & 11 & 10 \\
\hline & & 20 & 14 & 11 & 8 & 8 & 10 & 10 \\
\hline
\end{tabular}

The dispersion $\phi=0.48$ and the ratio of the size factor $w=1.1$ are estimated using edge $R$ package particularly for RNA-seq data.

Table 5: The sample sizes per group required for a balanced design in human breast cancer RNA-seq data. The sample sizes are calculated given the nominal FDR=0.10 and $80 \%$ power.

Table 5 reports the samples sizes under different scenarios including various minimum mean read counts in control condition $(1,5,10$ and 20$)$ and desired fold changes $(1.5,2,3$ and 4$)$ while controlling the FDR at 0.10 . We found that the original RNA-seq experiment [28] with a minimum sample size 31 in each condition can detect more than $80 \%$ of the prognostic genes at the FDR $(f=0.10)$ and $u_{0}=1$ if the desired fold change is 3 or more. Moreover, with a minimum sample size 42 in each condition, we found that it can detect more than $80 \%$ of the prognostic genes at the FDR $(f=0.10)$ and $u_{0}=5$ if the desired fold change is 2 or more.

\section{Discussion and Conclusion}

In this study, five methods $\left(n_{w 1}, n_{w 2}, n_{l w 1}, n_{l w 2}, n_{l r t}\right)$ were derived to calculate sample sizes using the Wald test and LRT statistics based on a negative binomial distribution for modeling an RNA-seq experiment. The parameters are estimated using the MLE and CMLE methods. Since the dispersion estimated from MLE has no closing form, it is difficult to derive the sample size formula. Therefore, all of the methods are based on a fixed and constant dispersion. A log-transformed approach corresponding to the modified $Z_{w 1}$ and $Z_{w 2}$ was used to derive two other test statistics $\left(Z_{l w 1}\right.$ and $\left.Z_{l w 2}\right)$. For all these statistical tests as well as the exact test [19] that are used to derive the sample size calculation formulas, gene expression levels are assumed to be independent in each sample. Although this assumption might not hold in reality, it is widely used in RNA-seq as well as in microRNA data analysis. In this study, we assume equal sample size in the two conditions to derive the sample size formula. The derived formula for sample size calculations can be easily extended to the unequal sample sizes by setting $n_{1}=\mathrm{k} n_{0}$. In our simulation study, we set the ratio of total size factors in two conditions as 1 and 1.2 instead of $w=2$ in the study [19]. In reality, the read depths of RNA-seq samples generated from the same run are very close to each other across conditions. Therefore, we think $w=1.2$ or close to 1 is more common than $w=2$. Furthermore, in our simulation and application studies, the minimum sample sizes required to achieve a nominal power of $80 \%$ with a predefined FDR $(f=0.05$ or 0.10$)$ are usually larger than those in an RNA-seq experiment due to the real costs. In such a situation, we can increase the read depth per sample to indirectly increase the mean of read counts $u_{0}$ in the control condition. Thus, the required sample sizes can be decreased correspondingly.

Among the methods we evaluated, the simulation results show that $n_{l w 2}$ from the log transformed Wald test with the parameters estimated from CMLE is the best method because a smaller sample size is required for designing an RNA-seq experiment while achieving a power close to or higher than $80 \%$ at a pre-defined FDR $=0.05$. The second best method is $n_{l w 1}$ with the parameters estimated from unrestricted MLEs. We also found that $n_{l r t}$ and $n_{\text {exact }}$ methods perform better than $n_{w 1}$ method based on the estimated power given the genes with a fold change $>2$. However, $n_{w 1}$ achieve a better power than $n_{l r t}$ and $n_{\text {exact }}$ given a fold change $\leq 2$. In summary, $n_{w 1}, n_{w 2}, n_{l r t}$ and $n_{\text {exact }}$ methods varied with different scenarios. Finally, since the log-transformed sample size calculation methods are more robust, simpler and require less time, we hope our tables can help and benefit for researchers and scientists in the design of RNA-seq experiments.

\section{Acknowledgments}

We thank Dr. Shyr and his coauthors for sharing their R codes with us that were used for comparison of our methods with theirs. This work was supported by P20GM103436 (N.G. Cooper, PI). Dr. Rai was supported by Wendell Cherry Chair in Clinical Trial Research in James Graham Brown Cancer Center (Dr. DM Miller, Director).

\section{References}

1. Ghosh A, Islam T (2016) Genome-wide analysis and expression profiling of glyoxalase gene families in soybean (Glycine max) indicate their development and abiotic stress specific response. BMC plant biology 16: 87.

2. Jiang Y, Malouf GG, Zhang J, Zheng X, Chen Y, et al. (2015) Long non-coding RNA profiling links subgroup classification of endometrioid endometrial carcinomas with trithorax and polycomb complex aberrations. Oncotarget 6: 39865-39876.

3. Peffers MJ, Liu X, Clegg PD (2014) Transcriptomic profiling of cartilage ageing. Genomics data 2: 27-28.

4. Robertson G, Schein J, Chiu R, Corbett R, Field M, et al. (2010) De novo assembly and analysis of RNA-seq data. Nature methods 7: $909-912$. 
Citation: Li X, Cooper NGF, Shyr Y, Wu D, Rouchka EC, et al. (2017) Inference and Sample Size Calculations Based on Statistical Tests in a Negative Binomial Distribution for Differential Gene Expression in RNA-seq Data. J Biom Biostat 8: 332. doi:10.4172/2155-6180.1000332

Page 12 of 12

5. Yue YJ, Liu JB, Yang M, Han JL, Guo TT, et al. (2015) De novo assembly and characterization of skin transcriptome using RNAseq in sheep (Ovis aries). Genetics and molecular research: GMR 14: 1371-1384.

6. Schliebner I, Becher R, Hempel M, Deising HB, Horbach R (2014) New gene models and alternative splicing in the maize pathogen Colletotrichum graminicola revealed by RNA-Seq analysis. BMC genomics 15: 842 .

7. Wen L, He K, Yang H, Ni Y, Zhang X, et al. (2008) Complete nucleotide sequence of a novel porcine circovirus-like agent and its infectivity in vitro. Science in China. Series C, Life sciences/Chinese Academy of Sciences 51: 453-458.

8. Craven KE, Gore J, Wilson JL, Korc M (2016) Angiogenic gene signature in human pancreatic cancer correlates with TGF-beta and inflammatory transcriptomes Oncotarget 7: 323-341.

9. van 't Veer LJ, Dai H, van de Vijver MJ, He YD, Hart AA, et al. (2002) Gene expression profiling predicts clinical outcome of breast cancer. Nature 415: 530-536.

10. Voelkerding KV, Lyon E (2010) Digital fetal aneuploidy diagnosis by next-generation sequencing. Clinical chemistry 56: 336-338.

11. Hansen KD, Wu Z, Irizarry RA, Leek JT (2011) Sequencing technology does not eliminate biological variability. Nature biotechnology 29: 572-573.

12. Auer PL, Doerge RW (2010) Statistical design and analysis of RNA sequencing data. Genetics 185: 405-416.

13. Fang Z, Cui X (2011) Design and validation issues in RNA-seq experiments. Briefings in bioinformatics 12: 280-287.

14. Marioni JC, Mason CE, Mane SM, Stephens M, Gilad Y (2008) RNA-seq: an assessment of technical reproducibility and comparison with gene expression arrays. Genome research 18: 1509-1517.

15. Wang ET, Sandberg R, Luo S, Khrebtukova I, Zhang L, et al. (2008) Alternative isoform regulation in human tissue transcriptomes. Nature 456: $470-476$.

16. Li Cl, Su PF, Guo Y, Shyr Y (2013) Sample size calculation for differential expression analysis of RNA-seq data under Poisson distribution. International journal of computational biology and drug design 6: 358-375.

17. Anders S, Huber W (2010) Differential expression analysis for sequence count data. Genome biology 11: R106.

18. Robinson MD, McCarthy DJ, Smyth GK (2010) edgeR: a Bioconductor package for differential expression analysis of digital gene expression data. Bioinformatics 26 : 139-140.

19. Li Cl, Su PF, Shyr Y (2013) Sample size calculation based on exact test for assessing differential expression analysis in RNA-seq data. BMC bioinformatics 14: 357.

20. Benjamini Y, Hochberg Y (1995) Controlling the False Discovery Rate: a Practical and Powerful Approach to Multiple Testing. J R Statist soc B 57: $289-300$.

21. Storey JD (2002) A direct approach to false discovery rates. JR Stat Soc Ser B 64: 479-498.

22. Robinson MD, Smyth GK (2008) Small-sample estimation of negative binomial dispersion, with applications to SAGE data. Biostatistics 9: 321-332.

23. Aban IB, Cutter GR, Mavinga N (2008) Inferences and Power Analysis Concerning Two Negative Binomial Distributions with An Application to MRI Lesion Counts Data. Computational statistics \& data analysis 53: 820-833.

24. Ng HK, Tang ML (2005) Testing the equality of two Poisson means using the rate ratio. Statistics in medicine 24: 955-965.

25. Gu K, Ng HK, Tang ML, Schucany WR (2008) Testing the ratio of two poisson rates. Biometrical journal. Biometrische Zeitschrift 50: $283-298$.

26. Thode HC (1997) Power and sample size requirements for tests of differences between two LPoisson rates. The Statistician 46: $227-230$.

27. Krishnamoorthy K, Thomson J (2004) A more powerful test for comparing two poisson means. J Stat Plan Infer 119: $23-35$.

28. Varley KE, Gertz J, Roberts BS, Davis NS, Bowling KM, et al. (2014) Recurrent read-through fusion transcripts in breast cancer. Breast cancer research and treatment 146: 287-297. 